\title{
Hypoxic Status Is Associated With The Intensity Of Hypoxia Inducible Factor (Hif)-1a Expression In A Premature Placenta
}

\author{
K. Kartini' ${ }^{1}$, M. Ekawati², Ani R. Prijanti ${ }^{3}$, Ahmad A. Jusuf ${ }^{3}$, Sri Widia A. Jusman ${ }^{4}$ \\ ${ }^{1}$ Departement of Histology, FMUSAKTI, Department of Histology, Faculty of Medicine, \\ Trisakti University J1. Kyai Tapa No 260 Grogol Jakarta 11440 Phone:+6221-5672731 \\ ${ }_{2}^{2}$ Doctor Program of Biomedical Science, FMUI \\ ${ }_{3}^{3}$ Departement of Biochemistry and Molecular Biology, FMUI \\ ${ }^{4}$ Departement of Histology, FMUI \\ Email : kartiniedwin@trisakti.ac.id \\ Receive : Mart 25th2020. Revised : Mart $5^{\text {th }}$ 2021. Published: June $5^{\text {th }} 2021$. \\ DOI : https://doi.org/10.22219/sm.Vol17.SMUMM1.11685
}

\begin{abstract}
Prematurity refers to live births before 37 weeks of gestation and associated with infant morbidity/mortality. Activation of HIF during the final pregnancy phase is believed to play a critical role in the pathogenesis of premature birth and other pregnancy disorders. This study aimed to analyze the relationship between hypoxicstatus and the intensity of HIF- $1 \alpha$ expression in a premature placenta.Stored biological materials premature placenta (paraffin blocks) was used in this study. Thirtyone samples of placental hypoxia $(\mathrm{H})$ and 28 samples of premature placental non-hypoxia $(\mathrm{N})$ as controls, were selected non-random consecutively. Subsequently, immunohistochemistry was performed to analyze HIF-1 $\alpha$ expression. TheChi-square testwas used to analyze the data and a p-value $<0.05$ was considered statistically significant.Moderate to strong intensity of HIF- $1 \alpha$ expressionwas observed in 58\% of hypoxic placenta samples, whereas most of nonhypoxic placental samples $(86 \%)$ did not expressed or expressed weaklyHIF-1 $\alpha$. There was a significant correlation between the intensity of HIF- $1 \alpha$ expression and placental hypoxia $(\mathrm{p}<0.05)$ and Odds Ratio $(\mathrm{OR})$ value was 8.31 with a $95 \%$ confidence interval $(2.32-29.77)$. The conclusion shows that hypoxic status is associated with intensity of hypoxia inducible factor $(\mathrm{HIF})-1 \alpha$ expression in a premature placenta.
\end{abstract}

Keywords : HIF-1 $\alpha$, hypoxia,placenta, premature.

\section{INTRODUCTION}

Prematurity is the birth of a live baby before 37 weeks' gestation, leading to inability to adapt to life outside the uterus due to its immature organ system. Premature birth events are associated with several risk factors, such as: history of previous preterm birth and abortion, anemia, high blood pressure, premature rupture of membranes, urinary tract infections, vaginal bleeding, twin pregnancy, oligohydramnios, preeclampsia, maternal age less than 20 years or more than 35 years old, less or no antenatal care, maternal smoking 
habits, increased levels of catecholamines in the mother's urine, and less than a year of pregnancy interval. The etiology of prematurity is still debatable.(Ahumada-Barrios and Alvarado, 2016)

Premature birth is closely related to infant morbidity and mortality.(Dong et al., 2012; Sulistiarini and Berliana, 2016) Premature babies will be at risk of developing health problems such as respiratory distress syndrome, retinopathy, breastfeeding disorders, necrotizing enterocolitis, infections, cerebral palsy, and can even cause infant death.(Gopagondanahalli et al., 2016) Premature birth can also cause milder disorders such as behavioral disorders, learning and language difficulties, attention disorders and hyperactivity, resulting in poor quality of human resources of future generations.(Sulistiarini and Berliana, 2016) The incidence of preterm birth continues to increase worldwide and according to WHO data the number of premature births is estimated at 15 million every year, and nearly one million children die from complications of preterm birth.(Sulistiarini and Berliana, 2016) WHO notes that the rate of premature events in Indonesia in 2010 was 15.5 per 100 live births.(Sulistiarini and Berliana, 2016) In the United States it is noted that preterm birth rates continue to increase, and among infants born very prematurely, more than half have cognitive, sensory, and behavioral deficits, and around 5-10\% experience motor deficits, including cerebral palsy.(Jellema et al., 2013; Ravarino et al., 2014) The state of hypoxia and tissue ischemia is likely to be the most common abnormality found in premature infant brain damage.(Ravarino et al., 2014)

In carrying out the process of cell development and maintaining a state of homeostasis as well as functioning properly, cells need oxygen availability continuously.(Vadlapatla, Vadlapudi and Mitra, 2013) Oxygen plays an important role in regulating membrane transport, intracellular signaling processes, cellular endurance, and expression of various genes. When tissue oxygenation demand increases beyond the availability of oxygen in the vascular system, hypoxia can occur, (Vadlapatla, Vadlapudi and Mitra, 2013) leading to the stabilization of a transcription factor known as hypoxiainducible factor-1 $\alpha$ (HIF-1 $\alpha$ ). Hypoxia-inducible factor $-1 \alpha$ is a subunit of HIF-1, regulating cellular homeostasis in response to hypoxic states.(Zaramellaet al., 2017) Hypoxia-inducible factor -1 was first discovered by Semenza, et al around 1991, in a study related to the erythropoietin gene (EPO).(Masoud and Li, 2015) In response to hypoxic states, the EPO gene is activated to transcribe in an effort to increase the production of red blood cells and oxygen-carrying capacity.(Vadlapatla, Vadlapudi and Mitra, 2013; Masoud and Li, 2015) This study found a DNA sequence that is important for the activation 
process of the EPO gene transcription called the hypoxia response element (HRE). Another study found that the activation of transcription of various regulatory genes was initiated by the binding of a specific protein induced in a state of hypoxia to HRE. This protein is identified as HIF-1.(Masoud and Li, 2015) Normal developments of the placenta and fetus depend on the HIF1- $\alpha$ modulation and cellular defense against oxidative stress.(Horii et al., 2016)

Placenta is responsible for distributing the feto-maternal circulation in terms of exchange of nutrients and metabolic waste. The welfare of the fetus in the womb depends on the placenta, that regulates the process of pregnancy and fetal growth and development in optimal conditions.(Salmani et al., 2014) A healthy placenta has a plasticity in adapting to environmental conditions thus facilitating the invasion of trophoblast and vascular remodeling. (Chakraborty, D. et al., 2016). The development and maturity of the placenta is a complex process and requires adequate blood supply so that the fetus can develop completely. In early pregnancy the placenta develops in an environment with low oxygen pressure (physiological hypoxia) in order to support placenta function.(Liu, Wang and Lin, 2014) All cells respond to hypoxic conditions by modifying gene expression.HIF is an important mediator in this process to facilitate placental vascularization. Additionally HIF activation during the late pregnancy phase is believed to play a critical role in the pathogenesis of IUGR, preeclampsia, premature birth or other pregnancy disorders.(Patel et al., 2010)

In preeclampsia placenta, over expression of HIF- $\alpha$ was thought to be an indicator of hypoxia state response. Study by Liu et al.showed a decrease in the expression of hypoxia-inducible factor prolyl 4-hydroxylase-1 (PHD-1) resulting an increased expression of HIF- $2 \alpha$, not HIF-1 $\alpha$ in placenta preeclampsia. Response to hypoxia would change the metabolism pattern of placenta cells so as to disrupt their growth. This study highlights the possibility that PHD and HIF could be potential targets for preeclampsia therapy. (Liu, Wang and Lin, 2014) Tal mentions that preeclampsia pathogenesis seems to be centered on the occurrence of placental hypoxia. This review also reports the increasing of cell-free HIF-1 $\alpha$ RNA expression in maternal plasma, which suffered from preeclampsia and IUGR. It can be thought that in preeclampsia, HIF-1 $\alpha$ becomes a molecular link between placental hypoxia and its downstream mediators and it is possible that HIF-1 $\alpha$ suppression or antagonism of its downstream mediators may serve as targets in preeclampsia therapy. (Tal, 2012) Futhermore, there have been no studies that analizing the relationship of hypoxia and HIF-1 $\alpha$ expression in premature placentas. This study aims to compare the condition of 
cellular hypoxia by assessing the expression of HIF-1 $\alpha$, between the placenta of hypoxic premature infants and those without hypoxia.

\section{METHODS}

Study design

This observational-analytical cross-sectional study was conducted in the Histology Laboratory, Department of Histology, Faculty of Medicine, University of Indonesia, from July to December 2014.

\section{Study samples}

Using stored biological material (SBM) of placenta in preterm infantsacross sectional comparative formula was applied to determine the large samples. The sample were selected nonconsecutively, resulting 31 placenta hypoxia and 28 placenta non-hypoxia as controls hypoxia based on assessment of blood gas analysis and / or pulse oxymetry of premature infants, if $\mathrm{PO}_{2}<30$ $\mathrm{mmHg}$ and / or $\mathrm{O}_{2}$ saturation $<60 \%$ ).

\section{Placental examination}

\section{Immunohistochemistry}

Samples for HIF-1 $\alpha$ immunohistochemical (IHC) staining weredeparafinized and rehydrated and subsequently incubated in endogenous peroxidase and protein blocks (Leica Biosystems, Newcastle) for 30 minutes. Sections then incubated in mice primary antibodies (anti human HIF-1 $\alpha$; dilution 1/100; Santa Cruz Biotechnology, Inc., Texas), at $4{ }^{\circ} \mathrm{C}$ overnight, then in post primary secondary antibodies (Rabbit anti Mouse IgG) and Polymer (Anti-Rabbit Poly-HRP$\mathrm{IgG}$ ) for 30 minutes each (Leica Biosystems, Newcastle). Detection with diaminobenzidine and hematoxylin was applied for 5 minutes, then dehydration process was conducted.IHC processing used positive controls (breast cancer) and negative controls.

The intensity of HIF-1 $\alpha$ expression was assessed in the syncytiotrophoblast and capillary endothelial cells nucleus with a scale: $(-)$ if not expressed; (+) if weak expression; (++) if moderate expression; and $(+++)$ when strongly expressed. The intensity scale was analyzed in a double blind manner with a kappa coefficient of 0.630 and $p<0.05$. Additionally, the sample was taken in several fields of view using microfoto (Optilab Professional Edition) with a serial objective magnification of $4 x, 10 x$, and $40 x$.

\section{Statistical analysis}

Statistical Product and Service Solution (SPSS) statistical program version 16.0 was used to analyze the data and Chi-square test was used to analyze the relationship between hypoxia with the intensity of HIF- $1 \alpha$ expression. A p-value $<0.05$ was considered statistically significant. 


\section{Ethical clearance}

This study was approved by the Committee of Health Research Ethics, Faculty of Medicine, University of Indonesia/Cipto Mangun kusumo Hospital, under no.506/H2.F1/ETIK/2014.

\section{RESULTS AND DISCUSSION}

HIF- $1 \alpha$ protein expressed moderate to strong in $58 \%$ of hypoxic placentas, whereas $86 \%$ of non-hypoxic placental group had no or weak HIF-1 $\alpha$ protein expression. This protein was observed in the syncytiotrophoblast and capillary endothelial cells nucleus. There was a significant relationship between hypoxic status and the intensity of HIF- $1 \alpha$ protein expression $(p<0.05)$ and Odds Ratio (OR) value was 8.31 with a 95\% confidence interval (2.32-29.77). (Figure 1, table 1). Our results showed an association between hypoxic status and HIF-1 $\alpha$ intensity, where a hypoxic placenta has 8.31 times more likely express HIF-1 $\alpha$ than non-hypoxic one.

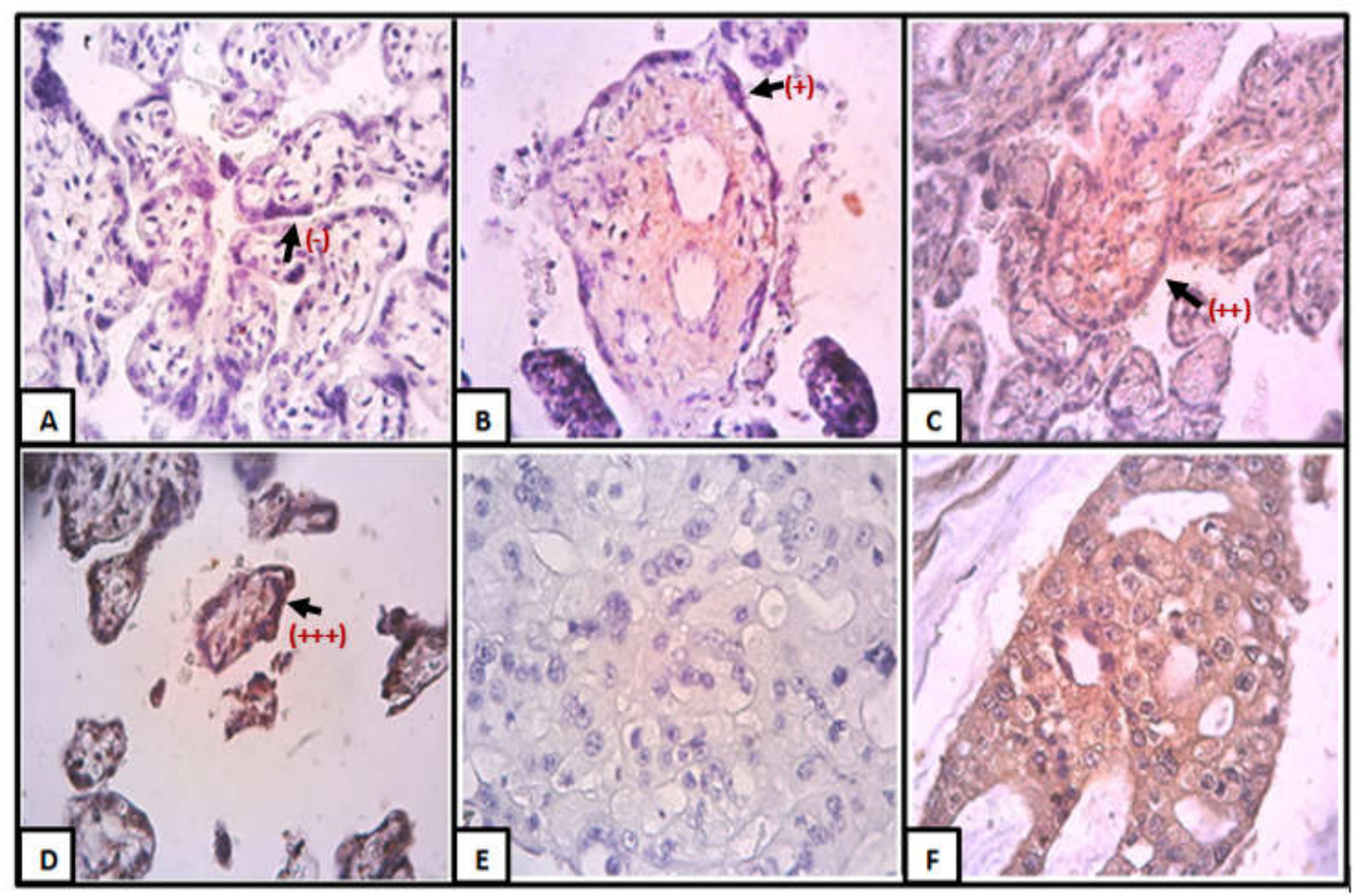

Figure 1. The intensity of HIF-1 $\alpha$ expression: [A]not expressed; [B]weak expression; [C]moderate expression; [D]strongly expressed; [E]Control(-); [F]Control(+) (breast cancer); IHC, 400x; counterstaining hematoxylin. 
Table 1. The relationship between hypoxia with the intensity of HIF-1 $\alpha$ expression

\begin{tabular}{|c|c|c|c|c|}
\hline \multirow{2}{*}{ Hypoxia status } & \multicolumn{2}{|c|}{ Intensity scale } & \multirow[t]{2}{*}{$\mathrm{p}$ value } & \multirow[t]{2}{*}{ OR (CI 95\%) } \\
\hline & moderate-strong & negative-weak & & \\
\hline Hypoxia & $18(58 \%)$ & $13(42 \%)$ & \multirow{2}{*}{$0.001 *$} & \multirow{2}{*}{$\begin{array}{c}8.31(2.32- \\
29.77)\end{array}$} \\
\hline Non-hypoxia & $4(14 \%)$ & $24(86 \%)$ & & \\
\hline
\end{tabular}

*Chi-squaretest

In the development of early pregnancy, hypoxia condition is needed for the process of cytotrophoblasts invasion and infiltration into the decidua, to maintain the feto-maternal circulation.Therefore, HIF-1 $\alpha$ will be expressed in the early trimester placenta and decreased with the increasing gestational age.(Dong et al., 2012; Rath et al., 2016)However, if the hypoxia continues until the final trimester, it can cause pain and even death for both mother and fetus. (Rath et al., 2016)Hypoxic placenta releases several mediators into the maternal circulation, one of which plays an important role is HIF-1 $\alpha$, a transcription factor for oxygen-dependent genes, to express proteins related to cell metabolism and angiogenesis.(Rath et al., 2016)HIF-1 $\alpha$ is a subunit of oxygensensitive HIF-1 transcription factors and this protein expression has increased in the placenta of pregnant women who experience complications of preeclampsia (PE),(Robb et al., 2017)and earlyonset preeclampsia pregnancies seems often leading to premature birth and fetal growth restriction.(Sriyantiet al., 2019)HIF-1 $\alpha$ regulate various cells and body systems in anoxic and ischemic conditions.(Lai and Liu, 2018) In premature placenta, we found an association between hypoxia status and HIF-1 $\alpha$ intensity, where a hypoxic placenta has 8.31 times more likely express HIF-1 $\alpha$ than non-hypoxic one. This may indicatedthat prolonged expression of HIF-1 and triggerpreterm birth.

Premature birth can occur idiopathically or spontaneously and is closely related to the process of infection and/or intra-uterine inflammation. The inflammatory process that occurs will induce oxidative stress due to an increase in proinflammatory cytokines and pertubed body's antioxidant system. This oxidative stress occurs in hypoxia circumstances.(Dong et al., 2012) Study by Dong et al. found that there was a significantly increased HIF- $1 \alpha \mathrm{mRNA}$ level in premature placenta compared with term placenta. This increased expression of HIF-1 $\alpha$ may be related to oxidative stress condition in the premature placenta.(Dong et al., 2012) Study by Jellema et al. has shown that inflammation of the cerebral tissue and mobilization of the peripheral innate immune system are in line with lesions and impaired function in the premature brain due to the state of global hypoxic ischemia. Further study related to cerebral involvement and systemic inflammatory processes due to global hypoxic ischemia is needed to discover a therapy of preterm infant encephalopathy.(Jellema et al., 2013) Study by Robb et al. found that the increased accumulation of HIF-1 $\alpha$ was associated with the occurrence of inflammatory-induced fetal growth restriction (FGR). FGR and preeclampsia often occur together and are associated with impaired uteroplacental perfusion and hypoxia in the placenta.(Robb et al., 2017)Study by Yuan et al. showed a significant overexpression of HIF-1 $\alpha$ in placenta patients with preeclampsia, leading to functional 
changes, including cell proliferation, migration and even constriction of the umbilical arteries, by affecting the expression of downstream molecules like 15-lippoxygenase (15-LO) and 15hydroxyeicosatetraenoic acid (15-HETE). (Yuan et al., 2014)

Our study found that HIF-1 $\alpha$ was observed in the syncytiotrophoblast and capillary endothelial cells nucleus.Rath et al.showed that HIF-1 $\alpha$ was expressed in the syncytiotrophoblast'snucleus and cytoplasm in preeclampsia placental tissue. The expression of this transcription factor was significantly different when compared with the controls placenta. (Rath et al., 2016)Similar with Lai et al., positive expression of HIF-1 $\alpha$ was observed in the nucleus and cytoplasm of syncytiotrophoblastas well as cytotrophoblast of the placental preeclampsia and its expression was significantly different compared with controls. Additionally, there was a higher HIF$1 \alpha$ expression severe preeclampsia placenta compared with the mildone.(Lai and Liu, 2018)Study by García-Honduvillaet al. found that the expression of both mRNA and HIF-1 $\alpha$ protein was increased in the placenta of women with venous insufficiency.(García-Honduvillaet al., 2018) The protein expression was found in the syncytio-cytotrophoblast layer and the endothelium capillary of corial villous. Venous insufficiency in the lower extremities is often a complication in pregnancy especially in the second trimester. The cause of venous insufficiency in pregnancy can be multifactorial. Progesterone contributes to reducing venous wall tone and the vasodilation effect of veins can increase the diameter of these vessels, thereby affecting the work of venous valves.(García-Honduvillaet al., 2018)In addition estrogen may induce water and sodium retention resulting in hypervolemia with consequent venous hypertension. Weaknesses in vascular walls can also be genetically inherited. This study illustrated the development of severe lesions with hypoxic patterns in the placenta of people with venous insufficiency. This may be related to abnormalities of the pelvic venous drainage system, including the uterus.(García-Honduvillaet al., 2018)Previous study also found that the existence of tissue hypoxia characterized by an increase in HIF gene and protein expression was associated with increased placental apoptosis and placental villi that undergoing hypoxia processes had microcalsification as consequences on cellular dynamics, and this calsificationseems to have an implication in events such oxidative stress and also changes in cellular metabolism.(Ortega et al., 2019)

Some abnormalities due to pregnancy complications, such as preeclampsia, low birth weight babies or premature births, can be caused by the impaired placental formation in early pregnancy, including disruption of the process of uterine spiral arterial remodeling and formation of corial villous vascularization. The increase in HIF in a hypoxic condition is associated with VEGF activity. (Ortega et al., 2019)Pathogenesis of placental formation disorders, especially in the process of angiogenesis involving vascular endothelial growth factor (VEGF) molecules. Study by Andraweera et al. found that VEGF m-RNA expression was decreased in complicated placenta (preeclampsia, low birth weight (LBW) and spontaneous preterm birth.(Andraweeraet al., 2012) The expression of VEGF mRNA and also protein depends on the presence of oxygen and will be 
increased in hypoxic states.It is believed that a placenta with abnormality such as preeclampsia has hypoxia,leading to an increase in VEGF expression. However, it is still debatable whether this low VEGF level is a consequence of decreased production or precisely as a result of inhibition by inhibitor molecules, for example sFLT1 (antiangiogenic placenta) which actually increases in the circulation of the mother's preeclampsia.(Andraweera et al., 2012)

Study by Abe et al.found that HIF-1 $1 \alpha$ stabilization or its translocation into the nucleus is influenced by nitric oxide (NO), leading to an increased expression of VEGF and iNOS mRNAs. Abe reported an increase in NO production in rat placenta during mid-pregnancy. Nitric oxide is a free radical in form of a water-soluble gas, which is a mediator of various cellular functions, including: smooth muscle relaxation, cell growth, and neurotransmission in the central nervous system.(Abe et al., 2013)NO regulates a number of cellular responses through cysteine Snitrosylation. S-nitrosylation process in Cys533 prevents the degradation process of HIF- $1 \alpha$ resulting in the accumulation of these transcription factors.(Abe et al., 2013)Study by Kartini et al. showed that fetal blood vessels appeared wide in some premature placenta, both in the hypoxia and non-hypoxia groups.(Kartini et al., 2015)This widening of the fetal blood vessels may be affected by NO. This study concluded that the hypoxia that occureds in premature placenta causes an increase in the number of fetal blood vessels as compensation for impaired oxygen homeostasis.(Kartini et al., 2015)An increase in the number of fetal blood vessels may be due to the activation of VEGF triggered by hypoxic conditions. Further study is needed to analyze the possibility of an increase in levels of VEGF and NO in premature human placenta.

In this study we found that $14 \%$ of non-hypoxic placentas expressed HIF-1 $\alpha$ moderate to strongly. Study by Kockar et al.stated that factors such as hormones and cytokines can induce the accumulation and activity of HIF-1 $\alpha$ in normoxic conditions.(Kockar, 2012) The presence of HIF$1 \alpha$ in some non-hypoxic premature placenta is likely a part of the physiological process to achieve homeostasis due to stress on placental tissue.

Many studies analyze hypoxia in placenta preeclampsia, and in this study we also found the occurrence of hypoxia condition in premature placenta. Until now the incidence of preterm birth is unpredictable, so prevention depends only on the health conditions and behavior of mothers in maintaining pregnancy. However, this can not prevent preterm labor due to its spontaneous occurrence and association with the state of hypoxia in the placenta.Previous study found that the expression of HIF-1 $\alpha$ at the gene and protein levels have increased in the premature placenta.(Dong et al., 2012)Thus, the results of our study are critical to initiatethedetectionof a molecular marker in preventing preterm birth, leading to the ultimate reduce of infant/child mortality and morbidity due to preterm birth complications. This study had limitations because no history of hypoxia has occurred in the placenta of premature infants due to the use of stored biological material. 


\section{CONCLUSIONS}

In our study, hypoxic status is associated with intensity of hypoxia inducible factor (HIF)$1 \alpha$ expression in the premature placenta.Hypoxic circumstances affect HIF-1 levels, as a cellular response to low oxygen environments, and inducing gene transcription such as VEGF, NO, etc.A further study is required to analyze the expression of the HIF-1 $\alpha$ gene and its hypoxia-related genes target, as a possibility of developing predictive marker in premature placenta, so the incidence of preterm birth can be reduce.

\section{AUTHORS CONTRIBUTION}

KK contributes to designing study methods, carrying out CPI processing, lab results analysis and statistical analysis; ME contributes to sample collection; ARP contributes to the supply of reagents for CPI; AAJ and SWJ contribute in designing study methods, lab results analysis and statistical analysis.

\section{CONFLICTS OF INTEREST}

There are no authors who declare a conflict of interest.

\section{ACKNOWLEDGEMENT}

Thank you to dr. Radiana D. Antarianto, M.Biomed, $\mathrm{PhD}$, who helped analyze the expression of HIF-1 $\alpha$ so that the examination was double blind. Thank you to the Research Council of the Faculty of Medicine, Universitas Trisakti, as well as those who have helped carry out this study.

\section{CONCLUSION}

The research conclusion is presented briefly, narrative and conceptual which describes the research findings and its impacts. Please avoid using bullets.

\section{REFERENCES}

Abe, H. et al. (2013) 'Nitric Oxide Induces Vascular Endothelial Growth Factor Expression in the Rat Placenta in Vivo and in Vitro', Bioscience Biotechnology and Biochemistry, 77(5), pp. 971-6. doi: 10.1271/bbb.120923.

Ahumada-Barrios, M. E. and Alvarado, G. F. (2016) 'Risk Factors for premature birth in a hospital', Revista Latino-Americana de Enfermagem, 24, pp. 1-8. doi: 10.1590/1518-8345.0775.2750.

Andraweera, P. H. et al. (2012) 'Placental expression of VEGF family mRNA in adverse pregnancy outcomes', Placenta, 33, pp. 467-72. doi: 10.1016/j.placenta.2012.02.013.

Chakraborty, D., et al. (2016) 'HIF-KDM3A-MMP12 regulatory circuit ensures trophoblast 
plasticity and placental adaptations to hypoxia', PNAS, 113(46), pp. E7212-E7221. doi: https://doi.org/10.1073/pnas.1612626113.

Dong, X. et al. (2012) 'The expression of thioredoxin-1 in preterm delivery placenta', Redox Report, 17(5), pp. 187-93. doi: 10.1179/1351000212y.0000000021.

García-Honduvilla, N. et al. (2018) 'Placentas from women with pregnancy-associated venous insufficiency show villi damage with evidence of hypoxic cellular stress', Human Pathology, 77, pp. 45-53. doi: 10.1016/j.humpath.2018.03.022.

Gopagondanahalli, K. R. et al. (2016) 'Preterm Hypoxic-Ischemic Encephalopathy', Frontiers in Pediatrics, 4(114), pp. 1-10. doi: 10.3389/fped.2016.00114.

Horii, M. et al. (2016) 'Human pluripotent stem cells as a model of trophoblast differentiation in both normal development and disease', Proceedings of the National Academy of Sciences of the United States of America, 113(27), pp. 3882-91. doi: 10.1073/pnas.1604747113.

Jellema, R. K. et al. (2013) 'Cerebral inflammation and mobilization of the peripheral immune system following global hypoxia-ischemia in preterm sheep', Journal of Neuroinflammation, 10(13), pp. 1-19. doi: 10.1186/1742-2094-10-13.

Kartini, K. et al. (2015) 'Fetal blood vessel count increases in compensation of hypoxia in premature placentas', UNIVERSA MEDICINA, 34(1), pp. 35-42. doi: 10.18051/univmed.2015.v34.035.

Kockar, F. (2012) 'Hypoxia and cytokines regulate carbonic anhydrase 9 expression in hepatocellular carcinoma cells in vitro', World Journal of Clinical Oncology, 3(6), pp. 82-91. doi: 10.5306/wjco.v3.i6.82.

Lai, H. and Liu, H. (2018) 'Expression and meaning analysis of HIF-1 $\alpha$ and HSP70 in preeclamptic placenta', Biomedical Research (India), 29(6), pp. 1240-43. doi: 10.4066/biomedicalresearch.29-17-3632.

Liu, W., Wang, S. J. and Lin, Q. De (2014) 'Study on the expressions of PHD and HIF in placentas from normal pregnant women and patients with preeclampsia', International Journal of Biological Sciences, 10(3), pp. 278-84. doi: 10.7150/ijbs.6375.

Masoud, G. N. and Li, W. (2015) 'HIF-1 $\alpha$ pathway: Role, regulation and intervention for cancer therapy', Acta Pharmaceutica Sinica B, pp. 378-89. doi: 10.1016/j.apsb.2015.05.007.

Ortega, M. A. et al. (2019) 'Upregulation of VEGF and PEDF in placentas of women with lower extremity venous insufficiency during pregnancy and its implication in villous calcification', BioMed Research International, 2019, p. 5320902. doi: 10.1155/2019/5320902.

Patel, J. et al. (2010) 'Regulation of Hypoxia Inducible Factors (HIF) in hypoxia and normoxia during placental development', Placenta, 31(11), pp. 951-957. doi: 10.1016/j.placenta.2010.08.008.

Rath, G. et al. (2016) 'HIF-1 Alpha and Placental Growth Factor in pregnancies complicated with preeclampsia: A qualitative and quantitative analysis', Journal of Clinical Laboratory Analysis, 30(1), pp. 75-83. doi: 10.1002/jcla.21819. 
Ravarino, A. et al. (2014) 'Cerebral hypoxia and ischemia in preterm infants the role of the clinical pathological dialogue in problem solving', Proceedings Journal of Pediatric and Neonatal Individualized Medicine $\bullet$, 3(2), pp. 1-9. doi: 10.7363/030272.

Robb, K. P. et al. (2017) 'Inflammation-induced fetal growth restriction in rats is associated with increased placental HIF-1 $\alpha$ accumulation', PLOS ONE, 12(4), pp. 1-15. doi: 10.1371/journal.pone.0175805.

Salmani, D. et al. (2014) 'Study of structural changes in placenta in pregnancy-induced hypertension.', Journal of natural science, biology, and medicine, 5(2), pp. 352-355. doi: 10.4103/0976-9668.136182.

Sriyanti, R. et al. (2019) 'The difference in maternal serum hypoxia-inducible factors- $1 \alpha$ levels between early onset and late-onset preeclampsia', Open Access Macedonian Journal of Medical Sciences, 7(13), pp. 2133-7. doi: 10.3889/oamjms.2019.601.

Sulistiarini, D. and Berliana, M. (2016) 'Faktor-faktor yang memengaruhi kelahiran prematur di Indonesia : analisis data Riskesdas 2013', E-Journal Widya Kesehatan dan Lingkungan, 1(2), pp. 109-115. doi: 10.1289/ehp.1002285.

Vadlapatla, R. K., Vadlapudi, A. D. and Mitra, A. K. (2013) 'Hypoxia-inducible factor-1 (HIF-1): a potential target for intervention in ocular neovascular diseases', Curr Drug Targets, 14(8), pp. 919-935. doi: 10.2174/13894501113149990015.

Yuan, D. et al. (2014) 'Enhancement of the HIF-1 $\alpha / 15-L O / 15-H E T E$ axis promotes hypoxiainduced endothelial proliferation in preeclamptic pregnancy', PLOS ONE, 9(5), p. e96510. doi: 10.1371/journal.pone.0096510.

Zaramella, P. et al. (2017) 'Hypoxia-Inducible Factor in cord blood of term and preterm newborns', J Ped Moth Care, 2(2), pp. 1-7. 